TAMKANG JOURNAL OF MATHEMATICS

Volume 41, Number 4, 361-373, Winter 2010

\title{
NONLOCAL CAUCHY PROBLEM FOR NONLINEAR MIXED INTEGRODIFFERENTIAL EQUATIONS
}

\author{
H. L. TIDKE AND M. B. DHAKNE
}

\begin{abstract}
The present paper investigates the existence and uniqueness of mild and strong solutions of a nonlinear mixed Volterra-Fredholm integrodifferential equation with nonlocal condition. The results obtained by using Schauder fixed point theorem and the theory of semigroups.
\end{abstract}

\section{Introduction}

Let $X$ be a Banach space with norm $\|\cdot\|$. Let $B=C\left(\left[t_{0}, t_{0}+\beta\right], X\right)$ be the Banach space of all continuous functions from $\left[t_{0}, t_{0}+\beta\right]$ into $X$ endowed with supremum norm

$$
\|x\|_{B}=\sup \left\{\|x(t)\|: t \in\left[t_{0}, t_{0}+\beta\right]\right\} .
$$

Consider the following nonlinear mixed Volterra-Fredholm integrodifferential equation of the form:

$$
\begin{gathered}
x^{\prime}(t)+A x(t)=f\left(t, x(t), \int_{t_{0}}^{t} k(t, s, x(s)) d s, \int_{t_{0}}^{t_{0}+\beta} h(t, s, x(s)) d s\right), t \in\left[t_{0}, t_{0}+\beta\right] \\
x\left(t_{0}\right)+g\left(t_{1}, t_{2}, \ldots, t_{p}, x(\cdot)\right)=x_{0},
\end{gathered}
$$

where $0 \leq t_{0}<t_{1}<t_{2}<\cdots<t_{p} \leq t_{0}+\beta,-A$ is the infinitesimal generator of a compact $C_{0}$ semigroup $T(t), t \geq 0$, in a Banach space $X$ and the nonlinear functions $f:\left[t_{0}, t_{0}+\beta\right] \times X \times X \times X \rightarrow X, g \in C\left(\left[t_{0}, t_{0}+\beta\right]^{p} \times X ; X\right), k, h:\left[t_{0}, t_{0}+\beta\right] \times\left[t_{0}, t_{0}+\right.$ $\beta] \times X \rightarrow X$ and $x_{0}$ is a given element of $X$. The symbol $g\left(t_{1}, t_{2}, \ldots, t_{p}, x(\cdot)\right)$ is used in the sense that in the place of '.' we can substitute only elements of the set $\left\{t_{1}, t_{2}, \ldots t_{p}\right\}$. For example $g\left(t_{1}, t_{2}, \ldots, t_{p}, x(\cdot)\right)$ may be given by

$$
g\left(t_{1}, t_{2}, \ldots, t_{p}, x(\cdot)\right)=\sum_{i=1}^{p} C_{i} x\left(t_{i}\right),
$$

Corresponding author: H. L. Tidke.

Received June 13, 2009; revised March 25, 2010.

2000 Mathematics Subject Classification. Primary 45N05; Secondary 47B38, 47H10.

Key words and phrases. Existence and uniqueness, mild and strong solutions, mixed VolterraFredholm integrodifferential equation, Schauder fixed point theorem, nonlocal condition. 
where $C_{i},(i=1,2, \ldots, p)$ are constants. In this case, (1.3) allows the measurements at $t=t_{0}, t_{1}, \ldots, t_{p}$, rather that just at $t=t_{0}$. So more information is available. This equations (1.1)-(1.3) can be applied in physics with better effect than equation (1.1) with classical initial condition.

The nonlocal condition, which is a generalization of the classical initial condition, was motivated by physical problems. The problem of existence of solutions of evolution equation with nonlocal conditions in Banach space was first studied by [6] and he investigated the existence and uniqueness of mild, strong and classical solutions of the nonlocal Cauchy problem. The study of differential and integrodifferential equations in abstract spaces with nonlocal condition has received much attention in recent years. We refer to the papers of Byszewski [6, 8, 9], Byszewski and V. Lakshmikantham [5], Balachandran and Ilamaran [2], K. Balachandran[3] and Xingmei Xue [16].

The objective of the present paper is to generalize the results of $[3,4,6,7,10,11,12$, $13,14]$. Here, we investigate the existence of mild and strong solutions of the problem (1.1)-(1.2). The main tool employed in our analysis is based on the Schauder fixed point theorem and semigroups theory.

The paper is organized as follows. In section 2, we present the preliminaries and the main results. Section 3 deals with proofs of the theorems. Finally in section 4 we give an example to illustrate the application of our results.

\section{Preliminaries and main results}

Before proceeding to the statement of our main results, we shall setforth some preliminaries and hypotheses that will be used in our subsequent discussion.

Definition 2.1. Let $\{T(t)\}_{t \geq 0}$ be a $C_{0}$ semigroup of operators on $X$. A $C_{0}$ semigroup $\{T(t)\}_{t \geq 0}$ is said to be a compact $C_{0}$ semigroup of operators on $X$ if $T(t)$ is a compact operator for every $t>0$.

In this paper we assume that $-A$ is the infinitesimal generator of a compact $C_{0}$ semigroup $\{T(t)\}_{t \geq 0}$ of operators on $X, D(A)$ is the domain of $A, t_{0} \geq 0$. We will need the following sets:

$$
B_{r}=\{x \in X, \quad\|x\| \leq r\} \quad \text { and } \quad E_{r}=\left\{z \in B, \quad\|z\|_{B} \leq r\right\},
$$

where $r>0$.

Definition 2.2. A continuous solution $x$ of the integral equation

$$
\begin{aligned}
x(t)= & T\left(t-t_{0}\right) x_{0}-T\left(t-t_{0}\right) g\left(t_{1}, t_{2}, \ldots, t_{p}, x(\cdot)\right) \\
& +\int_{t_{0}}^{t} T(t-s) f\left(s, x(s), \int_{t_{0}}^{s} k(s, \tau, x(\tau)) d \tau, \int_{t_{0}}^{t_{0}+\beta} h(s, \tau, x(\tau)) d \tau\right) d s, t \in\left[t_{0}, t_{0}+\beta\right]
\end{aligned}
$$

is said to be a mild solution of problem (1.1) $-(1.2)$ on $\left[t_{0}, t_{0}+\beta\right]$. 
Definition 2.3. A function $x$ is said to be a strong solution of problem (1.1)-(1.2) on $\left[t_{0}, t_{0}+\beta\right]$ if $x$ is differentiable almost everywhere on $\left[t_{0}, t_{0}+\beta\right], \quad x^{\prime} \in L^{1}\left(\left[t_{0}, t_{0}+\beta\right], X\right)$ and satisfying $(1.1)-(1.2)$ a.e. on $\left[t_{0}, t_{0}+\beta\right]$.

We need the following theorem for further discussion:

Theorem 2.4. (Schauder fixed point theorem [15], p-37) Let $S$ be a bounded, closed and convex subset of a Banach space $X$. If $f \in \mathcal{C}(S, S)$, where $\mathcal{C}(S, S)$ is the set of all compact maps from $S$ into $S$, then $f$ has at least one fixed point.

We list the following hypotheses for our convenience.

$\left(H_{1}\right)$ There exists a constant $G_{1}>0$ such that

$$
G_{1}=\max _{x \in E_{r}}\left\|g\left(t_{1}, t_{2}, \ldots, t_{p}, x(\cdot)\right)\right\| .
$$

$\left(H_{2}\right)-A$ is the infinitesimal generator of a compact $C_{0}$ semigroup $T(t), t \geq 0$ in $X$ such that

$$
\|T(t)\| \leq M
$$

for some $M \geq 1$.

$\left(H_{3}\right)$ There are constants $L_{1}, K_{1}$ and $H_{1}$ such that

$$
\begin{aligned}
L_{1} & =\max _{t_{0} \leq t \leq t_{0}+\beta}\|f(t, 0,0,0)\|, \\
K_{1} & =\max _{t_{0} \leq s \leq t \leq t_{0}+\beta}\|k(t, s, 0)\|, \\
H_{1} & =\max _{t_{0} \leq s \leq t \leq t_{0}+\beta}\|h(t, s, 0)\| .
\end{aligned}
$$

With these preparations, we are now in a position to state our main results to be proved in the present paper.

Theorem 2.5. Assume that

$\left(A_{1}\right)$ hypotheses $\left(H_{1}\right)-\left(H_{3}\right)$ hold,

$\left(A_{2}\right) f \in C\left(\left[t_{0}, t_{0}+\beta\right] \times X \times X \times X ; X\right)$ and there exists a constant $L>0$ such that

$$
\begin{aligned}
& \left\|f\left(t, x_{1}, y_{1}, z_{1}\right)-f\left(t, x_{2}, y_{2}, z_{2}\right)\right\| \leq L\left(\left\|x_{1}-x_{2}\right\|+\left\|y_{1}-y_{2}\right\|+\left\|z_{1}-z_{2}\right\|\right), \\
& \text { for } x_{i}, y_{i}, z_{i} \in X, i=1,2 \text { and } t \in\left[t_{0}, t_{0}+\beta\right] .
\end{aligned}
$$

$\left(A_{3}\right) k, h \in C\left(\left[t_{0}, t_{0}+\beta\right] \times\left[t_{0}, t_{0}+\beta\right] \times X ; X\right)$ and there exist constants $K, \quad H>0$ such that

$$
\left\|k\left(t, s, x_{1}\right)-k\left(t, s, x_{2}\right)\right\| \leq K\left\|x_{1}-x_{2}\right\|
$$

and

$$
\left\|h\left(t, s, x_{1}\right)-h\left(t, s, x_{2}\right)\right\| \leq H\left\|x_{1}-x_{2}\right\|
$$

for $x_{i}, y_{i} \in X, i=1,2$ and $t, s \in\left[t_{0}, t_{0}+\beta\right]$. 
$\left(A_{4}\right)$ The set

$$
\left\{x\left(t_{0}\right): x \in E_{r}, x\left(t_{0}\right)+g\left(t_{1}, t_{2}, \ldots, t_{p}, x(\cdot)\right)=x_{0}\right\}
$$

where

$$
\frac{M\left[\left\|x_{0}\right\|+G_{1}+L K_{1} \beta^{2}+L H_{1} \beta^{2}+L_{1} \beta\right]}{\left(1-M L\left[\beta+K \beta^{2}+H \beta^{2}\right]\right)} \leq r,
$$

with $M L\left[\beta+K \beta^{2}+H \beta^{2}\right]<1$, is precompact in $X$.

$\left(A_{5}\right)$ The function $g$ satisfies the following property

$g\left(t_{1}, t_{2}, \ldots, t_{p},\left(\lambda x_{1}+(1-\lambda) x_{2}\right)(\cdot)\right)=\lambda g\left(t_{1}, t_{2}, \ldots, t_{p}, x_{1}(\cdot)\right)+(1-\lambda) g\left(t_{1}, t_{2}, \ldots, t_{p}, x_{2}(\cdot)\right)$,

for $x_{i} \in E_{r},(i=1,2), \lambda \in(0,1)$.

Then problem (1.1)-(1.2) has a mild solution on $\left[t_{0}, t_{0}+\beta\right]$.

Theorem 2.6. Assume that

$\left(B_{1}\right)$ hypotheses $\left(H_{1}\right)-\left(H_{3}\right)$ hold,

$\left(B_{2}\right) X$ is a reflexive Banach space with norm $\|$.$\| and x_{0} \in D(A)$,

$\left(B_{3}\right) g\left(t_{1}, t_{2}, \ldots, t_{p}, x().\right) \in D(A)$,

$\left(B_{4}\right)$ There exists a constant $L>0$ such that

$\left\|f\left(t_{1}, x_{1}, y_{1}, z_{1}\right)-f\left(t_{2}, x_{2}, y_{2}, z_{2}\right)\right\| \leq L\left(\left|t_{1}-t_{2}\right|+\left\|x_{1}-x_{2}\right\|+\left\|y_{1}-y_{2}\right\|+\left\|z_{1}-z_{2}\right\|\right)$,

for $x_{i}, y_{i}, z_{i} \in X, i=1,2$ and $t_{1}, t_{2} \in\left[t_{0}, t_{0}+\beta\right]$.

$\left(B_{5}\right)$ There exist constants $K, H>0$ such that

$$
\left\|k\left(t_{1}, s, x_{1}\right)-k\left(t_{2}, s, x_{2}\right)\right\| \leq K\left(\left|t_{1}-t_{2}\right|+\left\|x_{1}-x_{2}\right\|\right)
$$

and

$$
\left\|h\left(t_{1}, s, x_{1}\right)-h\left(t_{2}, s, x_{2}\right)\right\| \leq H\left(\left|t_{1}-t_{2}\right|+\left\|x_{1}-x_{2}\right\|\right),
$$

for $x_{i}, y_{i} \in X, i=1,2$ and $t_{1}, t_{2} \in\left[t_{0}, t_{0}+\beta\right]$.

$\left(B_{6}\right)$ The set

$$
\left\{x\left(t_{0}\right): x \in E_{r}, x\left(t_{0}\right)+g\left(t_{1}, t_{2}, \ldots, t_{p}, x(\cdot)\right)=x_{0}\right\}
$$

where

$$
\frac{M\left[\left\|x_{0}\right\|+G_{1}+L K_{1} \beta^{2}+L H_{1} \beta^{2}+L_{1} \beta\right]}{\left(1-M L\left[\beta+K \beta^{2}+H \beta^{2}\right]\right)} \leq r,
$$

with $M L\left[\beta+K \beta^{2}+H \beta^{2}\right]<1$, is precompact in $X$. 
$\left(B_{7}\right)$ The function $g$ satisfies the following property

$$
\begin{aligned}
& g\left(t_{1}, t_{2}, \ldots, t_{p},\left(\lambda x_{1}+(1-\lambda) x_{2}\right)(\cdot)\right) \\
& \quad=\lambda g\left(t_{1}, t_{2}, \ldots, t_{p}, x_{1}(\cdot)\right)+(1-\lambda) g\left(t_{1}, t_{2}, \ldots, t_{p}, x_{2}(\cdot)\right),
\end{aligned}
$$

for $x_{i} \in E_{r},(i=1,2), \lambda \in(0,1)$.

$\left(B_{8}\right)$ The problem (1.1)-(1.2) has at most one mild solution.

Then $x$ is the unique strong solution of problem (1.1)-(1.2) on $\left[t_{0}, t_{0}+\beta\right]$.

Remark 2.7. We remark that K. Balachandran and M. Chandrasekaran in [1] have studied existence and uniqueness of local and global solutions of special forms of the equations (1.1) - (1.2) by using Banach fixed point theorem and analytic semigroup theory. Here our approach to the problem and conditions on functions are different from those in [1].

\section{Proofs of theorems}

\section{Proof of Theorem 2.5. Let}

$$
E=\left\{x \in B: x \in E_{r}, \quad x\left(t_{0}\right)+g\left(t_{1}, t_{2}, \ldots, t_{p}, x(\cdot)\right)=x_{0}\right\} .
$$

It is easy to see that $E$ is a bounded closed convex subset of $B$. We define a mapping $F: E \rightarrow E$ by

$$
\begin{aligned}
& (F x)(t)=T\left(t-t_{0}\right) x_{0}-T\left(t-t_{0}\right) g\left(t_{1}, t_{2}, \ldots, t_{p}, x(\cdot)\right) \\
& \quad+\int_{t_{0}}^{t} T(t-s) f\left(s, x(s), \int_{t_{0}}^{s} k(s, \tau, x(\tau)) d \tau, \int_{t_{0}}^{t_{0}+\beta} h(s, \tau, x(\tau)) d \tau\right) d s, t \in\left[t_{0}, t_{0}+\beta\right] .
\end{aligned}
$$

Since all the functions involved in the definition of the operator are continuous and $T(t)$ is $C_{0}$ semigroup operator, the operator $F$ is continuous. For $x \in E, t \in\left[t_{0}, t_{0}+\beta\right]$ and using hypotheses $\left(H_{1}\right)-\left(H_{3}\right)$ and assumptions $\left(A_{2}\right),\left(A_{3}\right)$, we have

$$
\begin{aligned}
\|(F x)(t)\| \leq & \left\|T\left(t-t_{0}\right) x_{0}\right\|+\left\|T\left(t-t_{0}\right) g\left(t_{1}, t_{2}, \ldots, t_{p}, x(\cdot)\right)\right\| \\
& +\left\|\int_{t_{0}}^{t} T(t-s) f\left(s, x(s), \int_{t_{0}}^{s} k(s, \tau, x(\tau)) d \tau, \int_{t_{0}}^{t_{0}+\beta} h(s, \tau, x(\tau)) d \tau\right) d s\right\| \\
\leq & M\left(\left\|x_{0}\right\|+G_{1}\right)+M \int_{t_{0}}^{t}\left[\| f\left(s, x(s), \int_{t_{0}}^{s} k(s, \tau, x(\tau)) d \tau,\right.\right. \\
& \left.\left.\quad \int_{t_{0}}^{t_{0}+\beta} h(s, \tau, x(\tau)) d \tau\right)-f(s, 0,0,0)\|+\| f(s, 0,0,0) \|\right] d s \\
\leq & M\left(\left\|x_{0}\right\|+G_{1}\right)
\end{aligned}
$$




$$
\begin{aligned}
& +M \int_{t_{0}}^{t}\left[L r+L \int_{t_{0}}^{s}\|k(s, \tau, x(\tau))-k(s, \tau, 0)+k(s, \tau, 0)\| d \tau\right. \\
& \left.+L \int_{t_{0}}^{t_{0}+\beta}\|h(s, \tau, x(\tau))-h(s, \tau, 0)+h(s, \tau, 0)\| d \tau+L_{1}\right] d s \\
\leq & M\left(\left\|x_{0}\right\|+G_{1}\right)+M \int_{t_{0}}^{t}\left[L r+L \beta\left(K r+K_{1}\right)+L \beta\left(H r+H_{1}\right)+L_{1}\right] d s \\
\leq & M\left[\left\|x_{0}\right\|+G_{1}+L r \beta+L K r \beta^{2}+L K_{1} \beta^{2}+L H r \beta^{2}+L H_{1} \beta^{2}+L_{1} \beta\right] \\
\leq & r .
\end{aligned}
$$

Thus, $F$ maps $E$ into itself and consequently $F \in C(E ; E)$.

Now, we prove that $F$ maps $E$ into a precompact subset $F(E)$ of $E$. For this purpose, we first show that the set $E(t)=\{(F x)(t): x \in E\}, t \in\left[t_{0}, t_{0}+\beta\right]$ is precompact in $X$. Observe that

$$
\begin{aligned}
E\left(t_{0}\right) & =\left\{(F x)\left(t_{0}\right): x \in E\right\} \\
& =\left\{x_{0}-g\left(t_{1}, t_{2}, \ldots, t_{p}, x(\cdot)\right): x \in E_{r}, \quad x\left(t_{0}\right)+g\left(t_{1}, t_{2}, \ldots, t_{p}, x(\cdot)\right)=x_{0}\right\} .
\end{aligned}
$$

Therefore, according to hypothesis $\left(A_{5}\right), E\left(t_{0}\right)$ is precompact in $X$.

Let $t>t_{0}$ be fixed. For an arbitrary $0<\epsilon<t-t_{0}$, we define a mapping

$$
\begin{aligned}
\left(F_{\epsilon} x\right)(t)= & T\left(t-t_{0}\right) x_{0}-T\left(t-t_{0}\right) g\left(t_{1}, t_{2}, \ldots, t_{p}, x(\cdot)\right) \\
& +\int_{t_{0}}^{t-\epsilon} T(t-s)\left[f\left(s, x(s), \int_{t_{0}}^{s} k(s, \tau, x(\tau)) d \tau, \int_{t_{0}}^{t_{0}+\beta} h(s, \tau, x(\tau)) d \tau\right)\right] d s .
\end{aligned}
$$

Since $T(t)$ is compact operator for every $t \geq 0$, then the set $E_{\epsilon}(t)=\left\{\left(F_{\epsilon} x\right)(t): x \in E\right\}$ is precompact in $X$ for every $\epsilon>0$. By using the equations (3.1), (3.3) and the hypotheses $\left(H_{2}\right)-\left(H_{3}\right)$ and assumptions $\left(A_{4}\right)-\left(A_{5}\right)$, we obtain

$$
\begin{aligned}
& \left\|(F x)(t)-\left(F_{\epsilon} x\right)(t)\right\| \\
& \leq \int_{t-\epsilon}^{t}\|T(t-s)\|\left\|f\left(s, x(s), \int_{t_{0}}^{s} k(s, \tau, x(\tau)) d \tau, \int_{t_{0}}^{t_{0}+\beta} h(s, \tau, x(\tau)) d \tau\right)\right\| d s \\
& \leq M \int_{t-\epsilon}^{t}\left[\| f\left(s, x(s), \int_{t_{0}}^{s} k(s, \tau, x(\tau)) d \tau,\right.\right. \\
& \left.\left.\quad \int_{t_{0}}^{t_{0}+\beta} h(s, \tau, x(\tau)) d \tau\right)-f(s, 0,0,0)\|+\| f(s, 0,0,0) \|\right] d s \\
& \leq M \int_{t-\epsilon}^{t}\left[L r+L \int_{t_{0}}^{s}\|k(s, \tau, x(\tau))-k(s, \tau, 0)+k(s, \tau, 0)\| d \tau\right. \\
& \left.\quad+L \int_{t_{0}}^{t_{0}+\beta}\|h(s, \tau, x(\tau))-h(s, \tau, 0)+h(s, \tau, 0)\| d \tau+L_{1}\right] d s
\end{aligned}
$$




$$
\begin{aligned}
& \leq M \int_{t-\epsilon}^{t}\left[L r+L \int_{t_{0}}^{s}(K\|x(\tau)-0\|+\|k(s, \tau, 0)\|) d \tau\right. \\
&\left.\quad+L \int_{t_{0}}^{t_{0}+\beta}(H\|x(\tau)-0\|+\|h(s, \tau, 0)\|) d \tau+L_{1}\right] d s \\
& \leq M \int_{t-\epsilon}^{t}\left[L r+L \beta\left(K r+K_{1}\right)+L \beta\left(H r+H_{1}\right)+L_{1}\right] d s \\
& \leq M\left[L r+L K r \beta+L K_{1} \beta+L H r \beta+L H_{1} \beta+L_{1}\right] \epsilon .
\end{aligned}
$$

This implies that there exist precompact sets arbitrary closed to the set $E(t)=\{(F x)(t)$ : $x \in E\}$. Hence, the set $\left\{(F x)(t): x \in E_{0}\right\}$ is precompact in $X$.

Next, we show that $F(E)$ is an uniformly equicontinuous family of functions. Let $t_{0}<s<t \leq t_{0}+\beta$. By using hypotheses $\left(H_{2}\right),\left(H_{3}\right)$ and assumptions $\left(A_{2}\right),\left(A_{3}\right)$, we have

$$
\begin{aligned}
&\|(F x)(t)-(F x)(s)\| \\
& \leq\left\|\left[T\left(t-t_{0}\right)-T\left(s-t_{0}\right)\right]\right\|\left(\left\|x_{0}\right\|+\left\|g\left(t_{1}, t_{2}, \ldots, t_{p}, x(\cdot)\right)\right\|\right) \\
&+\int_{t_{0}}^{s}\|[T(t-\tau)-T(s-\tau)]\| \\
& \times\left\|f\left(\tau, x(\tau), \int_{t_{0}}^{\tau} k(\tau, \sigma, x(\sigma)) d \sigma, \int_{t_{0}}^{t_{0}+\beta} h(\tau, \sigma, x(\sigma)) d \sigma\right)\right\| d \tau \\
&+\int_{s}^{t}\|T(t-\tau)\|\left\|f\left(\tau, x(\tau), \int_{t_{0}}^{\tau} k(\tau, \sigma, x(\sigma)) d \sigma, \int_{t_{0}}^{t_{0}+\beta} h(\tau, \sigma, x(\sigma)) d \sigma\right)\right\| d \tau \\
& \leq\left\|\left[T\left(t-t_{0}\right)-T\left(s-t_{0}\right)\right]\right\|\left(\left\|x_{0}\right\|+G_{1}\right)+\int_{t_{0}}^{s}\|[T(t-\tau)-T(s-\tau)]\| \\
& \times\left[L r+L K r \beta+L K_{1} \beta+L H r \beta+L H_{1} \beta+L_{1}\right] d \tau \\
&+M \int_{s}^{t}\left[L r+L K r \beta+L K_{1} \beta+L H r \beta+L H_{1} \beta+L_{1}\right] d \tau .
\end{aligned}
$$

Here we have proceeded as in the result (3.5). The right hand side of (3.5) is independent of $x \in E$ and tends to zero as $s \rightarrow t$ as a consequence of the continuity of $T(t)$ in the uniform operator topology for $t>0$, which follows from the compactness of $T(t), t>0$. Therefore, $F(E)$ is equicontinuous family of functions. Thus by Arzela-Ascoli's theorem, $F(E)$ is precompact. Hence by the Schauder fixed point theorem, $F$ has a fixed point in $E$ and any fixed point of $F$ is a mild solution of (1.1)-(1.2) on $\left[t_{0}, t_{0}+\beta\right]$. This completes the proof of the Theorem 2.5.

Proof of Theorem 2.6. All the assumptions of Theorem 2.5 are being satisfied, the problem (1.1) - (1.2) has a mild solution $x$ belonging to $E$. By the assumption $\left(B_{8}\right), x$ is the unique mild solution of the problem (1.1)-(1.2).

Now we will show that $x$ is unique strong solution of problem $(1.1)-(1.2)$ on $\left[t_{0}, t_{0}+\beta\right]$. 
Take

$$
\begin{aligned}
L_{2} & =\max _{t_{0} \leq t \leq t_{0}+\beta}\|f(t, x(t), 0,0)\|, \\
K_{2} & =\max _{t_{0} \leq s \leq t \leq t_{0}+\beta}\|k(t, s, x(s))\|, \\
H_{2} & =\max _{t_{0} \leq s \leq t \leq t_{0}+\beta}\|h(t, s, x(s))\| .
\end{aligned}
$$

For $0<\theta<t-t_{0}$ and $t \in\left[t_{0}, t_{0}+\beta\right]$, we have

$$
\begin{aligned}
x( & +\theta)-x(t) \\
= & {\left[T\left(t+\theta-t_{0}\right)-T\left(t-t_{0}\right)\right] x_{0} } \\
& -\left[T\left(t+\theta-t_{0}\right)-T\left(t-t_{0}\right)\right] g\left(t_{1}, t_{2}, \ldots, t_{p}, x(\cdot)\right) \\
& +\int_{t_{0}}^{t_{0}+\theta} T(t+\theta-s) f\left(s, x(s), \int_{t_{0}}^{s} k(s, \tau, x(\tau)) d \tau, \int_{t_{0}}^{t_{0}+\beta} h(s, \tau, x(\tau)) d \tau\right) d s \\
& +\int_{t_{0}+\theta}^{t+\theta} T(t+\theta-s) f\left(s, x(s), \int_{t_{0}}^{s} k(s, \tau, x(\tau)) d \tau, \int_{t_{0}}^{t_{0}+\beta} h(s, \tau, x(\tau)) d \tau\right) d s \\
& -\int_{t_{0}}^{t} T(t-s) f\left(s, x(s), \int_{t_{0}}^{s} k(s, \tau, x(\tau)) d \tau, \int_{t_{0}}^{t_{0}+\beta} h(s, \tau, x(\tau)) d \tau\right) d s \\
= & T\left(t-t_{0}\right)[T(\theta)-I] x_{0}-T\left(t-t_{0}\right)[T(\theta)-I] g\left(t_{1}, t_{2}, \ldots, t_{p}, x(\cdot)\right) \\
& +\int_{t_{0}}^{t_{0}+\theta} T(t+\theta-s)\left[f\left(s, x(s), \int_{t_{0}}^{s} k(s, \tau, x(\tau)) d \tau, \int_{t_{0}}^{t_{0}+\beta} h(s, \tau, x(\tau)) d \tau\right)\right. \\
& \quad-f(s, x(s), 0,0)+f(s, x(s), 0,0)] d s \\
& +\int_{t_{0}}^{t} T(t-s)\left[f\left(s+\theta, x(s+\theta), \int_{t_{0}}^{s+\theta} k(s+\theta, \tau, x(\tau)) d \tau, \int_{t_{0}}^{t_{0}+\beta} h(s+\theta, \tau, x(\tau)) d \tau\right)\right. \\
& \left.\quad-f\left(s, x(s), \int_{t_{0}}^{s} k(s, \tau, x(\tau)) d \tau, \int_{t_{0}}^{t_{0}+\beta} h(s, \tau, x(\tau)) d \tau\right)\right] d s .
\end{aligned}
$$

Using the assumptions of theorem, we have

$$
\begin{aligned}
& \|x(t+\theta)-x(t)\| \\
& \leq M \theta\left\|A x_{0}\right\|+M \theta\left\|A g\left(t_{1}, t_{2}, \ldots, t_{p}, x(\cdot)\right)\right\| \\
& \quad+\int_{t_{0}}^{t_{0}+\theta} M\left[\| f\left(s, x(s), \int_{t_{0}}^{s} k(s, \tau, x(\tau)) d \tau,\right.\right. \\
& \left.\left.\quad \int_{t_{0}}^{t_{0}+\beta} h(s, \tau, x(\tau)) d \tau\right)-f(s, x(s), 0,0)\|+\| f(s, x(s), 0,0) \|\right] d s \\
& \quad+\int_{t_{0}}^{t} M\left[\| f\left(s+\theta, x(s+\theta), \int_{t_{0}}^{s+\theta} k(s+\theta, \tau, x(\tau)) d \tau, \int_{t_{0}}^{t_{0}+\beta} h(s+\theta, \tau, x(\tau)) d \tau\right)\right.
\end{aligned}
$$




$$
\begin{aligned}
& \left.\quad f\left(s, x(s), \int_{t_{0}}^{s} k(s, \tau, x(\tau)) d \tau, \int_{t_{0}}^{t_{0}+\beta} h(s, \tau, x(\tau)) d \tau\right) \|\right] d s \\
& \leq M \theta\left\|A x_{0}\right\|+M \theta\left\|A g\left(t_{1}, t_{2}, \ldots, t_{p}, x(\cdot)\right)\right\|+\int_{t_{0}}^{t_{0}+\theta} M L\left[\int_{t_{0}}^{s} K_{2} d \tau+\int_{t_{0}}^{t_{0}+\beta} H_{2} d \tau\right] d s \\
& +M \int_{t_{0}}^{t_{0}+\theta} L_{2} d s+\int_{t_{0}}^{t} M L[\theta+\|x(s+\theta)-x(s)\| \\
& \quad+\int_{t_{0}}^{s} K(|s+\theta-s|+\|x(\tau)-x(\tau)\|) d \tau \\
& \left.\quad+\int_{s}^{s+\theta} K_{2} d \tau+\int_{t_{0}}^{t_{0}+\beta} H(|s+\theta-s|+\|x(\tau)-x(\tau)\|) d \tau\right] d s \\
& \leq M \theta\left\|A x_{0}\right\|+M \theta\left\|A g\left(t_{1}, t_{2}, \ldots, t_{p}, x(.)\right)\right\|+M L K_{2} \theta \beta+M L H_{2} \theta \beta \\
& +M L_{2} \theta+M L \theta \beta+M L \int_{t_{0}}^{t}\|x(s+\theta)-x(s)\| d s \\
& +M L K \theta \beta^{2}+M L K_{2} \theta \beta+M L H \theta \beta^{2} \\
& \leq P \theta+M L \int_{t_{0}}^{t}\|x(s+\theta)-x(s)\| d s,
\end{aligned}
$$

where

$$
\begin{aligned}
P= & M\left\|A x_{0}\right\|+M\left\|A g\left(t_{1}, t_{2}, \ldots, t_{p}, x(\cdot)\right)\right\|+M L K_{2} \beta+M L H_{2} \beta \\
& +M L_{2}+M L \beta+M L K \beta^{2}+M L H \beta^{2}+M L K_{2} \beta .
\end{aligned}
$$

Thanks to Gronwall's inequality, we get

$$
\|x(t+\theta)-x(t)\| \leq P \theta e^{M L \beta}, \quad \text { for } \quad t \in\left[t_{0}, t_{0}+\beta\right] .
$$

Therefore, $x$ is Lipschitz continuous on $\left[t_{0}, t_{0}+\beta\right]$. The Lipschitz continuity of $x$ on $\left[t_{0}, t_{0}+\beta\right]$ combined with $\left(B_{4}\right)$ and $\left(B_{5}\right)$ of Theorem 2.6 gives that

$$
t \rightarrow f\left(t, x(t), \int_{t_{0}}^{t} k(t, s, x(s)) d s, \int_{t_{0}}^{t_{0}+\beta} h(t, s, x(s)) d s\right)
$$

is Lipschitz continuous on $\left[t_{0}, t_{0}+\beta\right]$. By Corollary 4.11, from Chapter 4 of [13] we observe that the equation

$$
\begin{aligned}
y^{\prime}(t)+A y(t) & =f\left(t, x(t), \int_{t_{0}}^{t} k(t, s, x(s)) d s, \int_{t_{0}}^{t_{0}+\beta} h(t, s, x(s)) d s\right), \quad t \in\left[t_{0}, t_{0}+\beta\right] \\
y\left(t_{0}\right) & =x_{0}-g\left(t_{1}, t_{2}, \ldots, t_{p}, x(\cdot)\right)
\end{aligned}
$$

has a unique strong solution $y(t)$ on $\left[t_{0}, t_{0}+\beta\right]$ satisfying the equation

$$
y(t)=T\left(t-t_{0}\right) x_{0}-T\left(t-t_{0}\right) g\left(t_{1}, t_{2}, \ldots, t_{p}, x(\cdot)\right)
$$




$$
\begin{aligned}
& +\int_{t_{0}}^{t} T(t-s) f\left(s, x(s), \int_{t_{0}}^{s} k(s, \tau, x(\tau)) d \tau, \int_{t_{0}}^{t_{0}+\beta} h(s, \tau, x(\tau)) d \tau\right) d s \\
= & x(t), \quad t \in\left[t_{0}, t_{0}+\beta\right] .
\end{aligned}
$$

Consequently, $x(t)$ is the strong solution of initial value problem (1.1) $-(1.2)$ on $\left[t_{0}, t_{0}+\beta\right]$. This completes the proof of Theorem 2.6.

\section{Application}

In order to illustrate the applications of some of our main results, we consider the following nonlinear mixed Volterra- Fredholm partial integrodifferential equation of the form

$$
\begin{gathered}
w_{t}(u, t)-w_{u u}(u, t)=P\left(t, w(u, t), \int_{0}^{t} k_{1}(t, s, w(u, s)) d s, \int_{0}^{\beta} h_{1}(t, s, w(u, s)) d s\right) \\
0 \leq u \leq \pi \quad \text { and } \quad 0 \leq t \leq \beta
\end{gathered}
$$

with initial and boundary conditions

$$
\begin{aligned}
& w(0, t)=w(\pi, t)=0, \quad 0 \leq t \leq \beta, \\
& w(u, 0)+\sum_{i=1}^{p} w\left(u, t_{i}\right)=w_{0}(u), \quad 0<t_{1}<t_{2}<\cdots<t_{p} \leq \beta .
\end{aligned}
$$

where $P:[0, \beta] \times R \times R \times R \rightarrow R, k_{1}, h_{1}:[0, \beta] \times[0, \beta] \times R \rightarrow R$ are continuous functions. We assume that the functions $P, k_{1}$ and $h_{1}$ in (4.1)-(4.3) satisfy the following conditions:

1. There are constants $L_{1}^{*}, K_{1}^{*}, H_{1}^{*}$ and $G_{1}^{*}$ such that

$$
\begin{aligned}
L_{1}^{*} & =\max _{0 \leq t \leq \beta}|P(t, 0,0,0)|, \\
K_{1}^{*} & =\max _{t_{0} \leq s \leq t \leq t_{0}+\beta}\left|k_{1}(t, s, 0)\right|, \\
H_{1}^{*} & =\max _{t_{0} \leq s \leq t \leq t_{0}+\beta}\left|h_{1}(t, s, 0)\right|, \\
G_{1}^{*} & =\max \left\{\left|\sum_{i=1}^{p} w\left(u, t_{i}\right)\right|, \quad 0 \leq u<\leq \pi ; \quad x \in E_{r^{*}}\right\} .
\end{aligned}
$$

2. $-A$ is the infinitesimal generator of a compact $C_{0}$ semigroup $T(t), t \geq 0$ in $X$ such that

$$
\|T(t)\| \leq M^{*}
$$

for some $M^{*} \geq 1$. 
3. $P \in C([0, \beta] \times R \times R \times R ; R)$ and there exists a constant $L^{*}>0$ such that

$$
\left|P\left(t, x_{1}, y_{1}, z_{1}\right)-P\left(t, x_{2}, y_{2}, z_{2}\right)\right| \leq L^{*}\left(\left|x_{1}-x_{2}\right|+\left|y_{1}-y_{2}\right|+\left|z_{1}-z_{2}\right|\right),
$$

for $x_{i}, y_{i}, z_{i} \in R, i=1,2$.

4. $k_{1}, h_{1} \in C([0, \beta] \times[0, \beta] \times R ; R)$ and there exist respectively constants $K^{*}, H^{*}>0$ such that

$$
\left|k_{1}\left(t, s, x_{1}\right)-k_{1}\left(t, s, x_{2}\right)\right| \leq K^{*}\left|x_{1}-x_{2}\right|
$$

and

$$
\left|h_{1}\left(t, s, x_{1}\right)-h_{1}\left(t, s, x_{2}\right)\right| \leq H^{*}\left|x_{1}-x_{2}\right|,
$$

for $x_{i}, y_{i} \in R, i=1,2$.

5. The set

$$
\left\{w(u, 0): x \in E_{r^{*}}, w(u, 0)+\sum_{i=1}^{p} w\left(u, t_{i}\right)=w_{0}(u)\right\}
$$

where

$$
\frac{M^{*}\left[\left\|w_{0}(u)\right\|+G_{1}^{*}+L^{*} K_{1}^{*} \beta^{2}+L^{*} H_{1}^{*} \beta^{2}+L_{1}^{*} \beta\right]}{\left(1-M^{*} L^{*}\left[\beta+K^{*} \beta^{2}+H^{*} \beta^{2}\right]\right)} \leq r^{*},
$$

for all $u \in[0, \pi]$; with $M^{*} L^{*}\left[\beta+K^{*} \beta^{2}+H^{*} \beta^{2}\right]<1$, is precompact in $R$.

First, we reduce the equations (4.1)-(4.3) into (1.1) - (1.2) by making suitable choices of $A, f, g, k$ and $h$. Let $X=L^{2}[0, \pi]$. Define the operator $A: X \rightarrow X$ by $A z=-z^{\prime \prime}$ with domain $D(A)=\left\{z \in X: z, \quad z^{\prime}\right.$ are absolutely continuous, $z^{\prime \prime} \in X$ and $\left.z(0)=z(\pi)=0\right\}$. Then the operator $A$ can be written as

$$
A z=\sum_{n=1}^{\infty} n^{2}\left(z, z_{n}\right) z_{n}, \quad z \in D(A)
$$

where $z_{n}(u)=(\sqrt{2 / \pi}) \sin n u, n=1,2, \ldots$ is the orthogonal set of eigenvectors of $A$ and $A$ is the infinitesimal generator of a compact semigroup $T(t), t \geq 0$ and is given by

$$
T(t) z=\sum_{n=1}^{\infty} e^{-n^{2} t}\left(z, z_{n}\right) z_{n}, \quad z \in X
$$

Define the functions $f:[0, \beta] \times X \times X \times X \rightarrow X, \quad k:[0, \beta] \times[0, \beta] \times X \rightarrow X, \quad h:$ $[0, \beta] \times[0, \beta] \times X \rightarrow X$ and $g:[0, \beta]^{p} \times X \rightarrow X$ as follows

$$
\begin{aligned}
& f(t, x, y, z)(u)=P(t, x(u), y(u), z(u)), \\
& k(t, s, x)(u)=k_{1}(t, s, x(u)), \\
& h(t, s, x)(u)=h_{1}(t, s, x(u)), \quad \text { and }
\end{aligned}
$$




$$
g\left(t_{1}, t_{2}, \ldots, t_{p}, x(\cdot)\right)(u)=\sum_{i=1}^{p} w\left(u, t_{i}\right)
$$

for $t \in[0, \beta], x, y, z \in X$ and $0 \leq u \leq \pi$. Then the above problem (4.1)-(4.3) can be formulated abstractly as nonlinear mixed Volterra-Fredholm integrodifferential equation in Banach space $X$ :

$$
\begin{gathered}
x^{\prime}(t)+A x(t)=f\left(t, x(t), \int_{t_{0}}^{t} k(t, s, x(s)) d s, \int_{t_{0}}^{t_{0}+\beta} h(t, s, x(s)) d s\right), t \in\left[t_{0}, t_{0}+\beta\right] \\
x\left(t_{0}\right)+g\left(t_{1}, t_{2}, \ldots, t_{p}, x(\cdot)\right)=x_{0} .
\end{gathered}
$$

Since all the hypotheses of the Theorem 2.5 are satisfied, the Theorem 2.5 can be applied to guarantee the existence of a mild solution of the nonlinear mixed Volterra-Fredholm partial integrodifferential equations (4.1)-(4.3).

\section{Acknowledgement}

The authors are thankful to the referee for the improvement of the paper and the research work supported by North Maharashtra University, Jalgaon.

\section{References}

[1] K. Balachandran and M. Chandrasekaran, Existence of solutions of nonlinear integrodifferential equations with nonlocal condition, Journal of Applied Mathematics and Stochastic Analysis, 10(1997), 279-288.

[2] K. Balachandran and S. Ilamaran, Existence and uniqueness of mild and strong solutions of a Volterra integrodifferential equation with nonlocal conditions, Tamkang J. Math., 28 (1997), 93-100.

[3] K. Balachandran, Existence and uniqueness of mild and strong solutions of nonlinear integrodifferential equations with nonlocal condition, Differential Equations and Dynamical Systems, 6, 1/2(1998), 159-165.

[4] D. Bahuguna, Integrodifferential equations with analytic semigroups, Journal of Applied Mathematics and Stochastic Analysis, 16 (2003), 177-189.

[5] L. Byszewski and V. Lakshmikantham, Theorems about the existence and uniquess of solution of a nonlocal abstract Cauchy problem in a Banach space, Appl. Anal., 40(1990), 11-19.

[6] L. Byszewski, Theorems about the existence and uniquess of solutions of a semilinear evolution nonlocal Cauchy problem, J. Math. Anal. Appl., 162(1991), 494-505.

[7] L. Byszewski and Haydar Akca, On a mild solution of a semilinear functional-differential evolution nonlocal problem, Journal of Applied Mathematics and Stochastic Analysis, 10 (1997), 265-271.

[8] L. Byszewski, Application of properties of the right-hand sides of evolution equations to an investigation of nonlocal evolution problems, Nonlinear Analysis, 33 (1998), 413-426. 
[9] L. Byszewski, Existence of solutions of a semilinear functional-differential evolution nonlocal problem, Nonlinear Analysis, 34(1998), 65-72.

[10] M. B. Dhakne and G. B. Lamb, On global existence of solutions of nonlinear integrodifferential equations, Indian J. Pure. Appl. Math., 33 (2002), 665-676.

[11] M. B. Dhakne and S. D. Kendre, On Nonlinear Volterra integrodifferential equation in Banach Spaces, Mathematical Inequalities and Applications, 9, (2006), 725-735.

[12] M. Kanaraj, Existence of weak and strong solutions of an integrodifferential eqaution in Banach spaces, Proc. Indian Acad. Sci. (Math. Sci.), 108 (1998), 169-177.

[13] A. Pazy, Semigroups of Linear Operators and Applications to Partial Differential Equations, New York, Springer-Verlag, 1983.

[14] H. L. Tidke and M. B. Dhakne, On global existence of solutions of abstract nonlinear mixed integrodifferential equation with nonlocal condition, Communications on Applied Nonlinear Analysis, 16, (2009), 49-60.

[15] Gerald Teschl, Nonlinear Functional Analysis, Vienna, Austria, February 2001.

[16] Xingmei Xue, Existence of solutions for semilinear nonlocal Cauchy problems in Banach spaces, Electronic Journal of Differential Equations, 2005(2005), No. 64, 1-7.

Department of Mathematics, School of Mathematical Sciences, North Maharashtra University, Jalgaon-425 001, India.

E-mail: tharibhau@gmail.com

Department of Mathematics, Dr. Babasaheb Ambedkar Marathwada University, Aurangabad431004 , India.

E-mail: mbdhakne@yahoo.com 